Draft Version March 23, 2022

Preprint typeset using $\mathrm{AT}_{\mathrm{E} X} \mathrm{X}$ style emulateapj v. 08/22/09

\title{
AMIBA: SYSTEM PERFORMANCE
}

Kai-Yang Lin ${ }^{1,2}$, ChaO-Te Li ${ }^{1}$, Paul T.P. Ho ${ }^{1,3}$, Chin-Wei Locutus Huang ${ }^{2,4}$, Yu-Wei LiaO ${ }^{2,4}$, Guo-Chin Liu ${ }^{1,5}$, Patrick M. Koch ${ }^{1}$, Sandor M. Molnar ${ }^{1}$, Hiroaki Nishioka ${ }^{1}$, Keilchi Umetsu ${ }^{1,4}$, Fu-Cheng Wang ${ }^{2,4}$, Jiun-Huei Proty Wu ${ }^{2,4}$, Michael Kestevan ${ }^{6}$, Mark Birkinshaw ${ }^{7}$, Pablo Altamirano ${ }^{1}$, Chia-Hao Chang ${ }^{1}$, Shu-Hao Chang ${ }^{1}$, Su-Wei Chang ${ }^{1}$, Ming-Tang Chen ${ }^{1}$, Pierre Martin-Cocher ${ }^{1}$, Chin-Chiang Han ${ }^{1}$, Yau-De Huang ${ }^{1}$, Yuh-Jing Hwang $^{1}$, Fabiola Ibañez-Roman ${ }^{1}$, Homin Jiang ${ }^{1}$, Derek Y. Kubo ${ }^{1}$, Peter Oshiro ${ }^{1}$, Philippe Raffin ${ }^{1}$, Tashun Wei $^{1}$, Warwick Wilson ${ }^{6}$, Ke-Jung Chen ${ }^{1}$, And Tzinong Chiueh ${ }^{2,4}$

Draft version March 23, 2022

\begin{abstract}
The Y.T. Lee Array for Microwave Background Anisotropy (AMiBA) started scientific operation in early 2007. This work describes the optimization of the system performance for the measurements of the Sunyaev-Zel'dovich effect for six massive galaxy clusters at redshifts $0.09-0.32$. We achieved a point source sensitivity of $63 \pm 7 \mathrm{mJy}$ with the seven $0.6 \mathrm{~m}$ dishes in 1 hour of on-source integration in 2 -patch differencing observations. We measured and compensated for the delays between the antennas of our platform-mounted interferometer. Beam switching was used to cancel instrumental instabilities and ground pick up. Total power and phase stability were good on time scales of hours, and the system was shown to integrate down on equivalent timescales of 300 hours per baseline/correlation, or about 10 hours for the entire array. While the broadband correlator leads to good sensitivity, the small number of lags in the correlator resulted in poorly measured bandpass response. We corrected for this by using external calibrators (Jupiter and Saturn). Using Jupiter as the flux standard, we measured the disk brightness temperature of Saturn to be $149_{-12}^{+5} \mathrm{~K}$.

Subject headings: cosmic microwave background - galaxies: clusters: general — instrumentation: interferometers
\end{abstract}

\section{INTRODUCTION}

The angular power spectrum of cosmic microwave background (CMB) anisotropies carries a wealth of information on the physical processes in early epochs of the universe. A comparison of theoretical models with accurate measurements of CMB anisotropies thus constrains the fundamental cosmological parameters and models for cosmic structure formation. On larger angular scales, the temperature anisotropies are dominated by primary CMB fluctuations, whereas on smaller angular scales secondary effects such as the Sunyaev-Zel'dovich (SZ) effects due to galaxy clusters dominate over primordial anisotropies. The amplitude and location of the peak in the thermal SZ power spectrum are particularly sensitive to the amplitude of the primordial matter power spectrum, represented by the normalization $\sigma_{8}$, as well as the thermal history of the hot intracluster medium. The Cosmic Background Imager (CBI, Pearson et al. 2003) and Arcminute Cosmology Bolometer Array Receiver (ACBAR, Kuo et al. 2004) measured the CMB temperature power spectrum at large angular multipoles of $l \sim 3000$. While the CBI detected an excess

\footnotetext{
${ }^{1}$ Academia Sinica Institute of Astronomy and Astrophysics, P.O. Box 23-141, Taipei, Taiwan 106

2 Physics Department, National Taiwan University, Taipei, Taiwan 106

${ }^{3}$ Harvard-Smithsonian Center for Astrophysics, 60 Garden Street, Cambridge, MA 02138, USA

${ }^{4}$ Leung Center for Cosmology and Particle Astrophysics, National Taiwan University, Taipei, Taiwan 106

${ }^{5}$ Department of Physics, Tamkang University, 251-37 Tamsui, Taipei County, Taiwan

${ }^{6}$ Australia Telescope National Facility, Epping, NSW Australia 1710

${ }^{7}$ University of Bristol, Tyndall Avenue, Bristol BS8 1TL, UK
}

power over the theoretical prediction from the standard cosmological model, the ACBAR result has a larger error bar and is consistent with both an excess and no excess. To date the uncertainties of the high- $l$ measurements remain large. More accurate measurements on large angular scales around and beyond $l=3000$ are required to better constrain the value of $\sigma_{8}$ (e.g., Bond et al. 2005; Goldstein et al. 2003; Lin et al. 2004).

The Y.T. Lee Array for Microwave Background Anisotropy (AMiBA, Ho et al. 2009; Chen et al. 2009; Koch et al. 2009a) is designed to measure CMB anisotropies on these multipole scales. The AMiBA is located on the volcanic mountain Mauna Loa, Hawaii, at an altitude of $3400 \mathrm{~m}$. The array observes with a single sideband in $86-102 \mathrm{GHz}$, or at roughly $3 \mathrm{~mm}$ wavelength, with cooled HEMT low noise amplifiers (LNA). Each of the seven receivers measures two linear polarizations (X and $\mathrm{Y}$ ) and produces two corresponding IF channels (each $2-18 \mathrm{GHz}$ ). Out of the four possible cross-correlations with a pair of receivers, AMiBA employs a switching system to form either the $\left(\mathrm{XX}^{*}, \mathrm{YY}^{*}\right)$ or the $\left(\mathrm{XY}^{*}, \mathrm{YX}^{*}\right)$ product at the same time. Note that a circular polarizer is being developed so that AMiBA can choose to measure either the $\left(\mathrm{LL}^{*}, \mathrm{RR}^{*}\right)$ or the $\left(\mathrm{LR}^{*}\right.$, $\left.\mathrm{RL}^{*}\right)$ cross-correlations in the future. There are thus 21 baselines and 42 instantaneous correlations for the seven-element array. The correlation is further divided into complex visibilities in two frequency bands using an analog four-lag correlator (Li et al. 2004).

All antennas and receivers are mounted on a $6 \mathrm{~m}$ platform so that antennas can be closely packed without issues with shadowing and collision. In the 2007 and 2008 seasons, observations were made with $60 \mathrm{~cm}$ diameter dishes close-packed in the center of the platform. 
Wu et al. (2009) present details of the observations and analysis of six massive clusters.

In this paper we describe how the system performance was optimized for these targeted observations. Two companion papers discuss the data integrity (Nishioka et al. 2009) and the CMB and foreground uncertainty in the SZ flux estimation (Liu et al. 2009). Combined with published X-ray parameters, the SZ fluxes of six clusters were used to measure the Hubble parameter (Koch et al. $2009 \mathrm{~b}$ ) and to examine the scaling relations (Huang et al. 2009). Subaru weak lensing data for four of the clusters were analyzed with the SZ measurements to derive the baryon fraction (Umetsu et al. 2009).

This paper is organized as follows. Critical issues such as the noise temperatures, delay corrections, stability, spurious signal removal and characteristics of the correlators are described in $\$ 2$, 3 discusses the losses of the system, the calibration errors, and the integration of noise. Finally 4 summarizes our conclusions.

\section{OPTIMIZING INTERFEROMETER PERFORMANCE}

Prior to and during the 2007 observing season, commissioning activities identified parts of the operations which needed to be improved (Lin et al. 2009). In particular, Huang et al. (2008) reports on the deformation of the platform which can affect the performance of the interferometer. Fortunately, these platform errors are repeatable and can be modeled. Their effects on pointing, radio alignment, and phase errors are discussed in Koch et al. (2009a). For AMiBA operations in 2007-8 these effects were minimal. In this paper, we concentrate on other areas of the interferometer performance which were optimized.

\subsection{System Temperature}

To understand the gain stability of AMiBA, we first measured the receiver stabilities. The system temperature is monitored by a set of sky-dips in total power mode. The total power output from each IF channel can be approximated by

$$
\begin{aligned}
P_{I F}= & g k B\left[T_{r x}+T_{d i s h}+T_{c m b}\right. \\
& \left.+T_{a t m} / \sin (e l)+T_{g n d}(a z, e l)\right],
\end{aligned}
$$

where $g$ is the power gain, $k$ is the Boltzmann constant, $B$ is the bandwidth of each IF channel, and the $T$ 's denote the noise temperatures from the receiver $(r x)$, antenna (dish), CMB ( $\mathrm{cmb}$ ), the atmosphere ( $\mathrm{atm})$, and ground pickup $(g n d)$. A hot/cold load measurement is used to calibrate $g B$ and $T_{r x}$. The receiver noise temperatures are $55-75 \mathrm{~K}$ (Chen et al. 2009). Fitting the total power to $P=P_{0}+P_{1} / \sin (e l)$ lumps the contributions into skylike $\left(P_{1}\right)$ and receiver-like $\left(P_{0}\right)$ parts plus some residual contributions from the ground. The measurements show that the total receiver-like noise temperature is about $1 \sigma \sim 5 \mathrm{~K}$ higher than $T_{r x}$. The sky-like part is approximately $15 \mathrm{~K}$ at zenith in typical observing conditions. Including $T_{c m b}$, the system temperatures away from zenith are about $80-100 \mathrm{~K}$. Repeated hot/cold load measurements of the receiver noise temperatures show that $T_{r x}$ is stable within the measurement error $(\sim 5 \mathrm{~K})$. Hence, by monitoring the system temperature using sky-dips, we can reject inferior sky conditions and unstable instrument behavior.

\subsection{Delay Correction}

Since AMiBA is a coplanar array there is no fringe rotation in a tracking observation. Fringes occur when a source moves across the field of view (fov) creating a geometric delay. The fov of AMiBA equipped with 0.6$\mathrm{m}$ dishes is $23^{\prime}$ (Wu et al. 2009). The requirement on delay trimming is that the source delay should remain within the sampling range of the lag-correlator, which is $\pm 50 \mathrm{ps}$. As the source delay approaches the limit of sampling range, the error in the recovered visibility becomes larger with a consequent rapid drop in sensitivity. To allow a $2-\mathrm{m}$ baseline to observe a $23^{\prime}$ fov, which corresponds to a delay range of $\sim \pm 22 \mathrm{ps}$, the instrumental delay was specified to a tolerance of $\pm 20 \mathrm{ps}$.

To measure the delay for each correlation, all dishes were removed and a noise source was mounted between receivers (e.g. Ant 1 and $\mathrm{Ant}_{2}$ ). A fringe is generated when the noise source moves from Ant 1 toward $\mathrm{Ant}_{2}$, simulating a fringe due to a celestial source.

$L\left(x, \tau_{a}\right)=\mathcal{R}\left(\int_{I F} d f \mathbb{R}^{\prime}(f) e^{-i 2 \pi\left[\left(f+f_{L O}\right) \frac{2 x}{c}+f\left(\tau_{2}-\tau_{1}+\tau_{a}\right)\right]}\right)$,

where $x$ is the displacement of noise source, $f$ is the IF frequency, and $\mathbb{R}^{\prime}$ is the complex response function of the baseline excluding the linear part of the phase due to lags $\left(\tau_{a}, a=1 \ldots 4\right)$ in the correlator. $\mathcal{R}$ takes the real part of the expression and is done implicitly whenever necessary hereafter. $\tau_{1}$ and $\tau_{2}$ represent the instrumental delays in

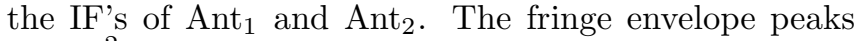
when $\frac{2 x}{c}=\tau_{1}-\tau_{2}-\tau_{a}$. The relative delay $\tau_{1}-\tau_{2}$ is measured with respect to the central lag (with $\tau_{a}=0$ ). Equation (2) is usually referred to as the lag output or the lag data throughout this work.

We found the instrumental delays for all IF channels using relative delay measurements. Short cables were then inserted into each IF for compensation. After this trimming procedure the residual delays were measured by fitting fringes for the Sun without the dishes, modeling the fringes as the convolution of the observed point source fringe with a circular disk. The differences between observation and model are consistent with residual delays of \pm 15 ps (RMS). Except for the delays due to platform deformation, the delays between antennas were therefore well controlled.

\subsection{Bandpass Shape Measurement}

The AMiBA correlator has four lags and outputs two spectral bands to cover the $2-18 \mathrm{GHz}$ band. Knowing the bandpass shape is an important aspect of obtaining good visibilities using this type of correlator (see next section $₫ 2.4$. Because the analog correlator contributes significantly to the bandpass shape, we adopted a baseline-based measurement approach. The Fourier transform of the fringe $L\left(x, \tau_{a}\right)$ against $x$ is used to determine $\mathbb{R}^{\prime}$ for each baseline, with a spectral sampling of about $0.8 \mathrm{GHz}$. Each lag output is transformed independently. Fig. 11 displays the gain and phase responses of all valid measurements after the four lag outputs are averaged together. Averaging the phase responses of the four lags, the delays are canceled leaving only the common mode of the spectral variation. The gain responses of the four lags are summed and then normalized such 
that the averaged gain between $2-18 \mathrm{GHz}$ is set to 1 .

The conversion from observed fringe rate to the $\mathrm{RF}$ frequency is proportional to the noise source translation speed. We believe a $\pm 1 \%$ jitter is present in the translation stage we used, which introduced roughly $\pm 1 \mathrm{GHz}$ uncertainty in the response frequency. This causes one of the major problem in developing an accurate visibility extraction method from our lag data (see \$2.4). An improved measurement setup involving simultaneous injection of a single frequency source is being developed to achieve a higher accuracy.

The effective bandwidths, defined as $B=$ $\left|\int d f \mathbb{R}^{\prime}\right|^{2} / \int d f\left|\mathbb{R}^{\prime}\right|^{2}$, are insensitive to the uncertainty in the response frequency. Based on our bandpass measurements, the effective bandwidths of the AMiBA correlators are calculated and shown in Fig.2. They generally fall in the range of $7-13 \mathrm{GHz}$.

\subsection{Extracting the Interferometer Visibilities}

Several approaches can be used to convert the four measured lags of the AMiBA correlator into complex visibilities in two bands over the $16 \mathrm{GHz}$ bandwidth. We find that the inaccuracies inherent in this inversion need to be corrected by external calibration. Here we adopt the formalism of Wu et al. (2009) (see Li et al. 2004, for an alternative formalism). The lag output in equation (2) can be expressed in matrix form as $L_{a}=\mathbb{R}_{a k} \mathbb{V}_{k}^{s r c}$, where $\mathbb{V}_{k}^{s r c}$ is the source visibility. Subscript $a$ indexes the $N_{\text {lag }}=4$ lags, and subscript $k$ indexes the $N_{f}$ discretized frequency samples $f_{k}$, where $N_{k}$ is usually much larger than $N_{\text {lag }}$.

The transformation relies on a kernel $\mathbb{K}_{a k}$, which is an estimate of the response matrix $\mathbb{R}_{a k}$. The kernel is integrated in frequency into two bands $\overline{\mathbb{K}}_{a c}$, where $c=1 \ldots 4$ indexes the real and imaginary parts of the two bands. We use the inverse of the integrated kernel to construct the raw visibility $\mathbb{V}_{c}^{\text {raw }} \equiv \overline{\mathbb{K}}^{-1}{ }_{c a} L_{a}$.

Ideally we would like $\mathbb{K}_{a k}=\mathbb{R}_{a k}$ so that $\mathbb{V}_{c}^{\text {raw }}$ is closest to $\mathbb{V}_{c}^{s r c}$. However, when $\mathbb{K}_{a k}$ is an inaccurate representation of $\mathbb{R}_{a k}$, due to measurement errors, variations with temperature or time, insufficient spectral resolution in the measurement, or insufficient information about the response, errors in visibilities occur. Fig. 3] demonstrates the calculation of raw visibility using simulated drift scans in three cases when (1) the kernel is the exact response, (2) there are measurement errors, and (3) there is no knowledge about the response. The correct visibility should appear as a Gaussian in amplitude with a linearly increasing phase. It can be seen that case (1) recovers the correct result, whereas deviations from this form increase with decreasing accuracy of the kernel. We therefore must obtain a calibrated visibility from the raw visibility $\mathbb{V}_{b}^{c a l} \equiv C_{b c} \mathbb{V}_{c}^{r a w}$, where $b$ has the same index range as $c$, and $C_{b c}$ is the calibration matrix, which can be obtained by comparing the raw visibility of a planet (the calibrator) to the theoretical visibility.

In the analysis of data taken in 2007 and 2008, the flat kernel (right-most function in Fig. 3) was assumed, and planet calibrations were applied. We have estimated the errors introduced by external calibration by running simulations on point source models. This error is on the order of $\pm 2 \%(1 \sigma)$ in the absolute fluxes with no detectable bias. This is small compared to the thermal noise and the measurement errors on the planet itself.

\subsection{Stability}

The stability of the system was examined by measuring the variation in visibilities for a few bright planets during local times $8 \mathrm{pm}$ to $8 \mathrm{am}$, as normally used for observing. For this test, the ephemerides of the planets were taken at the beginning of each track but not updated during the observation. This causes a pointing error that increases to about an arcminute over 12 hours. To account for this, two sets of visibility data for each planet were chosen as calibrating events. A linear interpolation was used to remove the linear drift. For data without bracketing events, the nearest calibration was used. Fig. 4 shows an example of a stability measurement. The gain stability was found to have an RMS variation around 5\%, and the phase to have an RMS variation around $0.1 \mathrm{rad}$. The measurements also reveal that the phase response is more sensitive to changes in environment than the gain response, especially in the first hour after shelter opening.

Fig 5 plots the flux of Jupiter recovered from the data set used in Fig 4. Data was calibrated by the first measurement at UT $12 \mathrm{~h}$ (not plotted). The recovered flux varied within $\pm 4 \%$ of the expected flux until sunrise. Calculation of the calibrator flux is discussed in $\$ 3.2$

Based on the stability measurements, we chose to use a calibration interval of two to three hours, to give calibrations good to about $5 \%$ in gain and $0.1 \mathrm{rad}$ in phase for each baseline. Calibration requires $\sim 10 \%$ of telescope observing time.

\subsection{Minimizing Instrumental and Ground Pickup}

The signal in the lag output should be constant when AMiBA tracks a source. However, the weak signal we measure is susceptible to slowly-varing contamination. The system is designed with a phase switching and demodulation scheme to remove contamination such as a common mode leakage in the IF paths. To modulate the signal, we use a PIN switch to change the LO between two carefully adjusted delay lines, and thus changes the phase of IF signal by $180^{\circ}$. The demodulation is done in the readout process. The aim was to remove contamination between the down-conversion mixer and the correlator readout.

However, mixers in the correlator can pick up higherorder signals such as $\left|E_{1}\right|^{2}\left|E_{2}\right|^{2}$ in addition to their nominal output, which is proportional to $E_{1} E_{2}^{*}$ or $|E|^{2}$, where $E_{i}$ stands for the voltage from $\mathrm{Ant}_{i}$. If the power of the IF signal is modulated by the phase switching pattern, then this higher order response can generate an output that is coherent with the demodulation pattern and becomes a spurious signal. This effect is, indeed, seen in AMiBA, where phase switching of the LO can result in a power difference as large as $0.3 \mathrm{~dB}$. The LO power modulation is carried through to the IF in varying amounts depending on the mean LO power level at the mixer. The IF modulation can be undetectable for optimally-tuned mixers, but is up to $3 \mathrm{~dB}$ for under-pumped mixers.

To reduce the IF modulation, the LO drive level is optimized for minimum conversion loss for each mixer. Modulation of the LO power would then have the least impact on the IF power. Furthermore, to reduce the 

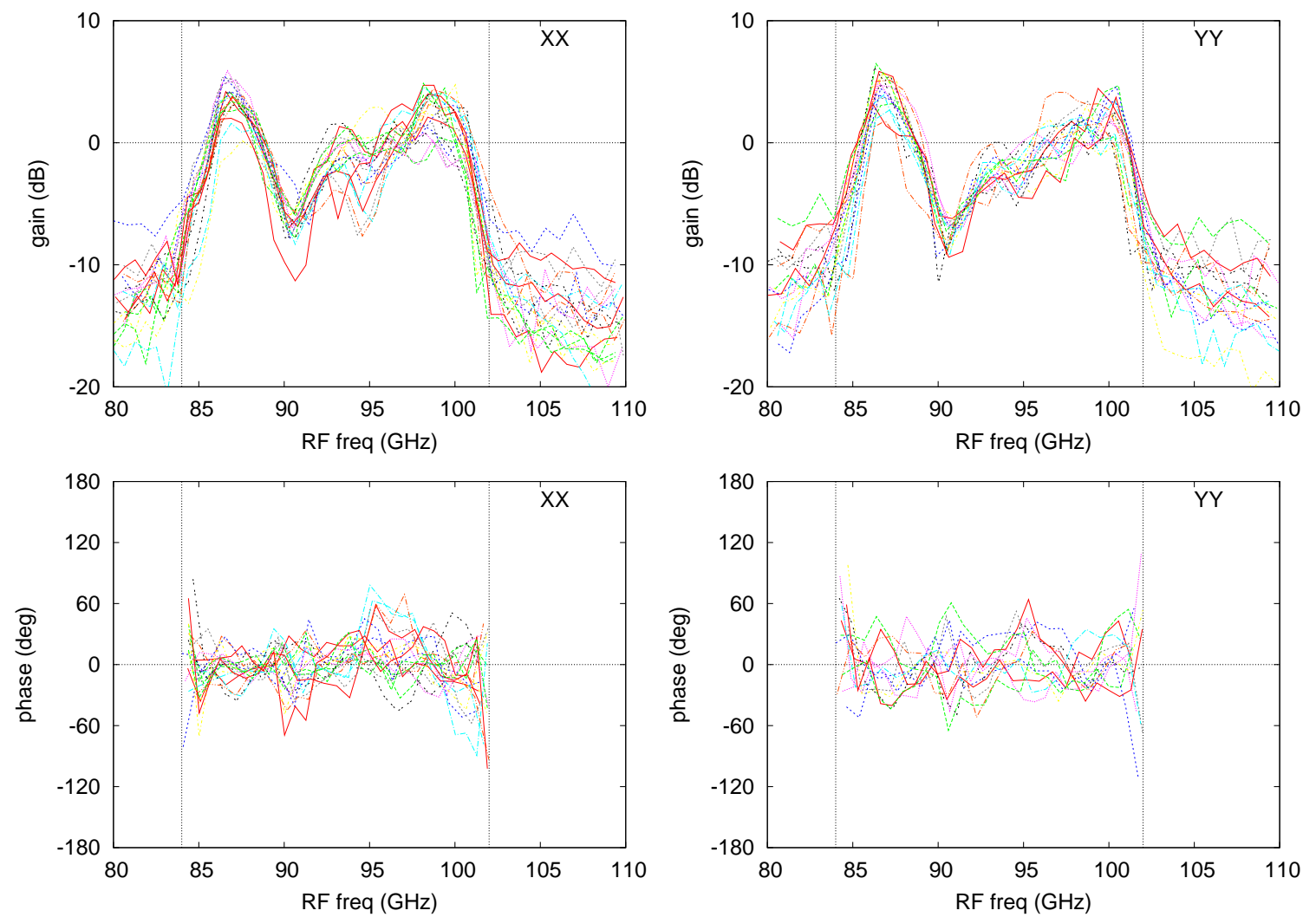

FIG. 1. - The complex responses of AMiBA. Responses include effects from the RF components, IF components, and the analog correlator. Top and bottom panels display the gain and phase responses respectively. Each line represents one cross-correlation (XX on the left and YY on the right) of a pair of receivers. Vertical dashed lines indicate RF frequencies of 84 and $102 \mathrm{GHz}$ (IF frequencies $0 \mathrm{and} 18 \mathrm{GHz}$ ).



Fig. 2.- Effective bandwidths of the AMiBA correlators calculated from the bandpasses displayed in Fig 1 The percentages are based on a nominal input bandwidth of $16 \mathrm{GHz}$.

drifting of LO level with ambient temperature changes, the final amplifier and frequency doubler in the LO chain are operated in the soft saturation regime. Additional protection is provided by temperature controls installed before the 2009 observing season.

Spurious signals external to the system, such as ground pickup, will still affect the data. We used a subtraction scheme similar to the one used by CBI (Padin et al. 2002) to suppress the slowly-varying signals. In practice, we have found that the spurious signal in individual patches in $\sim 5$ hrs integration can be as high as $\pm 7 \mathrm{Jy} /$ beam, but that after subtraction a cluster with brightness $0.3 \mathrm{Jy}$ can be detected at $9 \sigma$ level in 11 hours (i.e., with 5.5 hours on-source integration). The observing strategy and the data analysis are given in Wu et al. (2009).

\section{ACHIEVED SYSTEM PERFORMANCE}

\subsection{Overall Efficiency}

For each baseline, losses include the antenna loss, antenna misalignment, and the correlation loss. The antenna loss is mainly related to our Cassegrain design. Koch et al. (2009c) calculate the overall antenna efficiency to be 0.58 for the $1.2 \mathrm{~m}$ and $60 \mathrm{~cm}$ dishes alike. The loss originates mainly from three factors: the illumination efficiency, the secondary blockage, and the forward spillover. The AMiBA feeds provide a Gaussian illumination pattern and the the reflectors are designed to have a $-10.5 \mathrm{~dB}$ edge taper. Compared to an uniformly illuminated reflector, only $90 \%$ of the dish is effectively used. The shadow of the secondary mirror blocks about $8 \%$ of the collecting area, giving a loss of 0.92 . The edge of the secondary corresponds to the edge of the primary mirror. Therefore the illumination is either reflected by the primary to the sky or is emitted toward the sky direcly. The latter part constitutes about $22 \%$ of the energy giving the forward spillover factor of 0.78 . We favored a design with slightly worse forward spillover but little to none backward spillover (illumination toward the ground) to reduce system temperature.

The antenna misalignment consists of the mechanical installation error and the dynamical deformation of the platform. The former error was measured to be around $3^{\prime}$ during the 2007 observing season (Wu et al. 2009, in preparation) and will be improved for future observations. The latter error was inferred from photogramme- 



FIG. 3.- The upper two rows display three complex response functions. From the left, two slightly different functions are taken from the measurements shown in Fig. 1 with all four lags plotted explicitly. The third is an assumed flat response function. We use the left-most function to simulate a set of fringes from a flat spectrum source moving along the baseline. Then we follow the lag-to-visibility procedures in $\$ 2.4$ to obtain the bottom three sets of complex visibilities using each of the response functions as the transformation kernel. The horizontal axis is the source offset presented as drift time. To the left we see the case when the kernel is the same as the response function. The central plots show the results with small errors in the kernel. On the right we show the result of using an assumed flat kernel. Even though the deviation from ideal behavior can be as large as $50 \%$ in visibilities, calibration can correct for the errors.

try measurements of the platform surface to be less than $1^{\prime}$ (Koch et al. 2009a). The two errors together attenuate the primary beam by $2 \%$. Antenna misalignment may also cause pointing errors for some baselines. This effect is not considered in individual baseline efficiencies but will be considered in the array efficiency. There is also a loss of efficiency from the noise contributed by the rejected correlations in the analog correlator. The estimated correlation efficiency from this effect is 0.81 .

When combining baselines from the entire array, pointing error and system stability also lower the efficiency by degrading the coherence of signal from different measurements. The pointing error is less than $0.4^{\prime}$ (Koch et al. 2009a) and decreases the efficiency by less than $2 \%$. The large alignment error, on the other hand, contributes as much as $12 \%$ loss in the 2007 and 2008 observations. As described in $\$ 2.5$, the system stability is approximately $\pm 5 \%$ in gain and \pm 0.1 rad in phase. Taken over all baselines this results in a reduction of signal by about $2 \%$. Table 1 summarizes the major losses in the system.
TABLE 1

Summary of Losses of The System

\begin{tabular}{ll}
\hline \hline \multicolumn{1}{c}{ Systematics } & \multicolumn{1}{c}{ Efficiency } \\
\hline Antenna illumination $^{\mathrm{a}}$ & 0.90 \\
Antenna blockage $^{\mathrm{a}}$ & 0.92 \\
Antenna spillover $^{\mathrm{a}}$ & 0.78 \\
Antenna others effects $^{\mathrm{a}, \mathrm{b}}$ & 0.90 \\
Antenna total $_{\text {Alignment }^{\mathrm{b}}}$ & $0.90 \times 0.92 \times 0.78 \times 0.90=0.58$ \\
Correlation $^{\mathrm{a}}$ & 0.98 \\
Overall Baseline & 0.81 \\
Deformation/Pointing $^{\mathrm{b}}$ & $0.58 \times 0.98 \times 0.81=0.46$ \\
Stability $^{\mathrm{b}}$ & 0.98 \\
Overall Array & $0.46 \times 0.88 \times 0.98=0.40$ \\
\hline
\end{tabular}

a The facor is based on theoretical calculation.

b The facor is derived from measured quantities.

The baseline efficiency has been checked by comparing the signal-to-noise ratio (SNR) of Jupiter's fringe to the ratio of Jupiter's antenna temperature and the system 

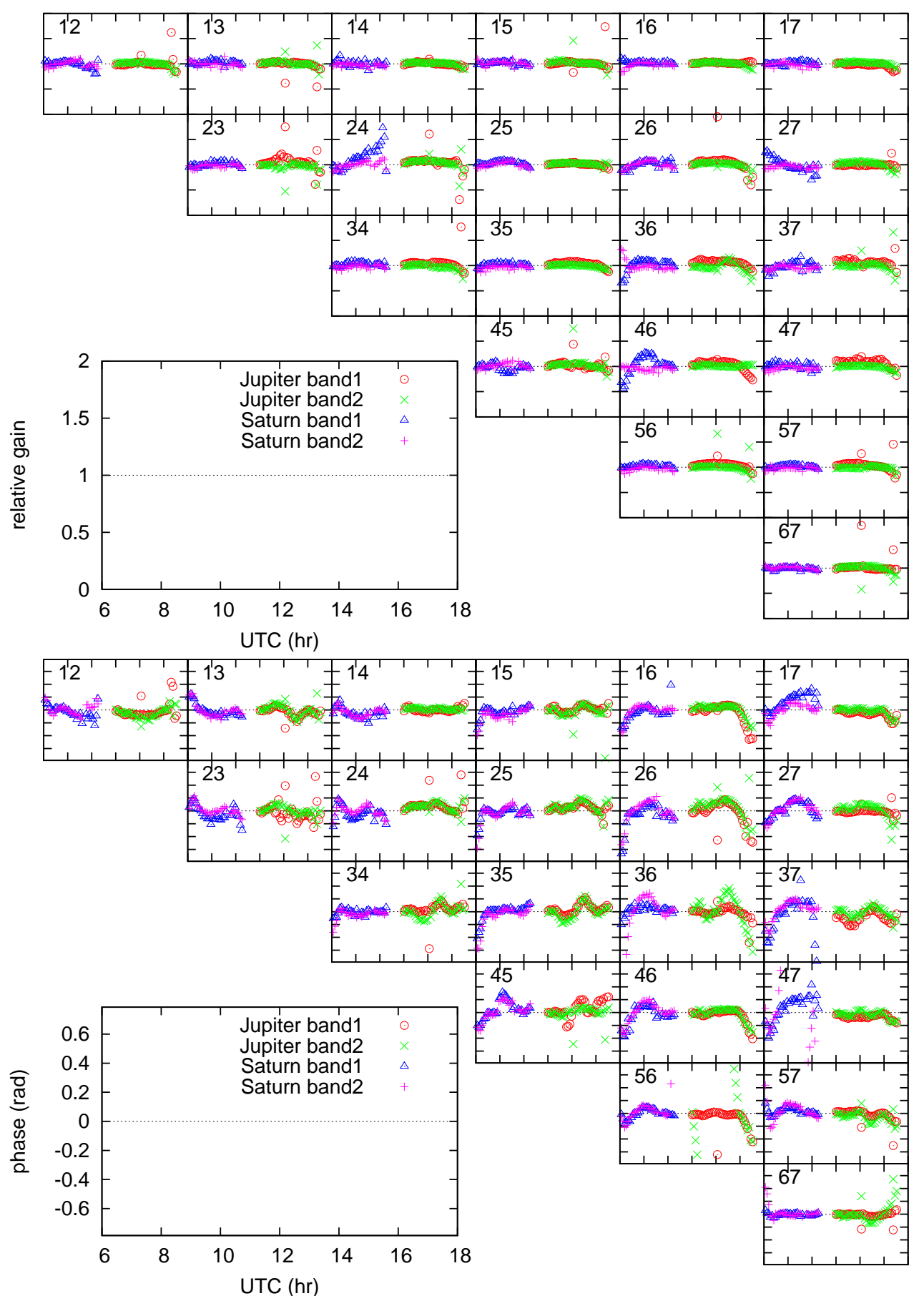

FIG. 4.- Visibilities recovered from a repeated two-patch tracking of Saturn (open triangles and plus symbols) and Jupiter (open circles and cross symbols) in the local time range $8 \mathrm{pm}$ to $8 \mathrm{am}$ (UT $6 \mathrm{hr}$ to $18 \mathrm{hr}$ ). The upper panel shows the relative gain fluctuation, and the lower panel shows the phase variation. Both are shown for the XX correlations. The number in the upper-left corner of each subplot indicates the antenna combination. The scale of the plot and the UT time range are indicated at the bottom left subplot of each panel.

temperature. $\eta_{b l}=\operatorname{SNR}_{J u p} /\left(\frac{T_{a, J u p}}{T_{\text {sys }}} \sqrt{B_{\text {eff } \frac{t_{\text {rec }}}{2}}}\right)$, where $T_{a, J u p}$ is the antenna temperature of Jupiter, typically around $0.1 \mathrm{~K}$ for the $60 \mathrm{~cm}$ dishes, and $\mathrm{SNR}_{J u p}$ is the SNR of Jupiter under the corresponding recording time $t_{r e c}$ $(=0.452$ sec currently). An average effective bandwidth of $B_{\text {eff }} \sim 10 \mathrm{GHz}$ was assumed in the calculation. The measured efficiency scatters from 0.2 to 0.5 with an error bar of approximately 0.2 . The error originates mainly from the noise estimation of the signal-dominant fringe, the occasional large readout noise, and also the variation of effective bandwidth. The overall array efficiency will be covered in 3.3 .

\subsection{Calibrator}

The raw visibilities recovered from the lag data have the systematic losses discussed above and are further affected by instrumental delay, gain drift, phase variation as well as the imperfect lag-to-visibility transformation. We calibrate visibilities by interspersed two-patch observations of a planet. This converts our visibility ampli- 


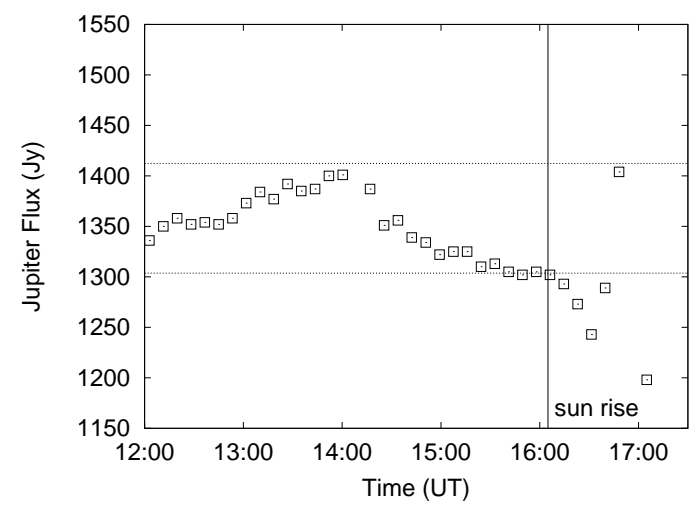

FIG. 5.- Jupiter flux recovered from the stability measurements in Fig 4 Two horizontal dotted lines indicate $\pm 4 \%$ of the expected Jupiter flux.

tudes to flux density units and references the phase to the calibrating planet position.

Taking planet data with the subtraction scheme, and applying the same calibration scheme used for cluster data (one calibration about every three hours), we find that the recovered peak flux in the image domain shows an RMS scatter of about $3 \%$.

The flux densities of the planets are calculated from published disk brightness temperatures and the apparent angular sizes assuming a black-body spectrum. We adopt the values: Jupiter $171.8 \pm 1.7 \mathrm{~K}$ (Page et al. 2003 ; Griffin et al. 1986), Saturn 149.3 $\pm 4.1 \mathrm{~K}$ (Ulich 1981), and Mars 206.8 $\pm 5.6 \mathrm{~K}$ (Ulich 1981). Fig 6 shows the recovered flux of the main calibrators (Jupiter and Saturn) in the 2007 and 2008 observations and the expected flux from calculation. For the phase reference and flux standard we use the first Jupiter measurement on each night when Jupiter is observed, as this provides the best calibration for the earlier Saturn measurements. The scatter of the Jupiter flux densities agrees with the scatter in one night as shown in Fig.5. The flux density of Saturn is systematically lower than the calculated value by approximately $5 \%$. It was verified that the lower flux was not the result of an error in flux standard. This was checked by artificially setting the phase error to zero in the calibrated visibilities of Saturn and forming an image. The recovered flux density displays no systematic offset from the calculated level, to within the error bound. The apparent deficit in the flux density for Saturn is likely due to insufficient phase calibration in the 2007 data, when calibrations more than two hours apart were often used. Note that the effect of Saturn's ring is not included in the calculation of Saturn's flux density. The ring inclination was about -15 deg for the 2007 observations and about -10 deg for the 2008 observations. We estimate that our flux density scale is good to about $\pm 5 \%$ in absolute terms. Based on Jupiter's flux scale, our measurement of Saturn's disk brightness temperature is $149_{-12}^{+5} \mathrm{~K}$.

A final note about calibration is on the difference of spectra between the calibrator and the SZ effect. Our primary calibrators, Jupiter and Saturn, are dominated by thermal emission near $94 \mathrm{GHz}$. The black-body spectrum favors slightly higher frequency than does the SZE spectrum. Regardless of the choice of assumed passband shape, the difference of effective central frequency, de-

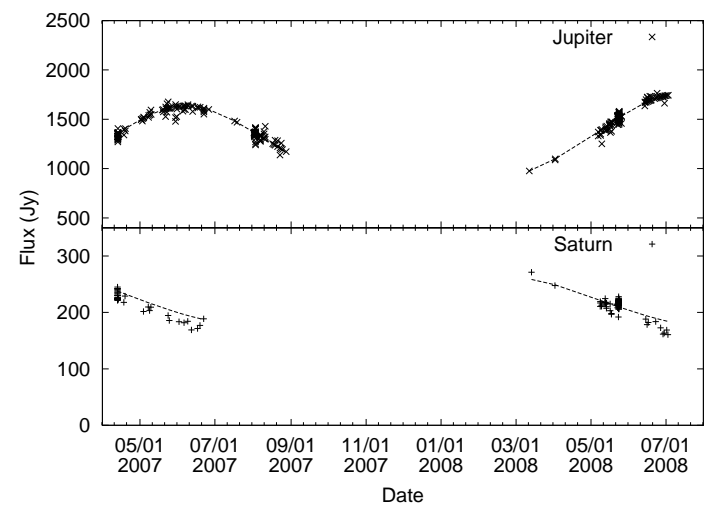

FIG. 6. - The recovered flux densities of our main calibrators, Jupiter (plus symbols) and Saturn (cross symbols) from the observations in 2007 and 2008. Data were calibrated by the first Jupiter measurement in the same night, or the nearest Jupiter measurement. The expected flux densities are plotted as dashed and solid lines for Jupiter and Saturn respectively. Note that Saturn's ring was not taken into account in the flux density estimation.

fined by $f_{c}=\left[\int_{\text {band }} R(f) S(f) f d f\right] /\left[\int_{\text {band }} R(f) S(f) d f\right]$ with $S(f)$ being the source spectrum and $R(f)$ being the passband, is less than $1.5 \%$ of the bandwidth. Its effect on the calibration is thus negligible compared to other errors.

\subsection{Noise Integration}

Based on the signal-to-noise ratio (SNR) of Jupiter's fringe and the discussion in 3.1 we find that the array has an overall efficiency of about 0.4. The improvement in sensitivity with integration time depends critically on the removal of spurious signals using the subtraction scheme and subsequent data flagging. To verify the sensitivity, we examine the variation of signal and noise in the reconstructed map with integration time in Figure 7 The integration time here refers to the accumulation of observing time spent in each individual visibility band. For example, when the telescope tracks a source for 3 minutes, the total integration time for 21 baselines, 2 polarizations, and 2 bands is $t_{\text {tot }}=180 \mathrm{sec} \times 21 \times 2 \times 2=15120 \mathrm{sec}$. Since the visibilities are used with non-uniform weights in forming the image, we calculate an effective integration time, which for 3 minutes on-source integration is defined as $t_{\text {eff }}=\frac{\left(\sum_{i} w_{i}\right)^{2}}{\sum_{i} w_{i}{ }^{2}} \times 180 \mathrm{sec}$, where $w_{i}$ denotes the weighting given to each data set. In this analysis, a natural weighting is adopted.

Since the noise comes from two patches and the signal comes from only one, the point source sensitivity can be estimated by $\sigma=\frac{2 k T_{s y s}}{\eta_{a l l} A_{p h y s}} \frac{1}{\sqrt{t_{e f f} B_{c h}}}$, where $T_{\text {sys }}=$ $100 \mathrm{~K}, B_{c h}=5 \mathrm{GHz}, \eta_{\text {all }}$ is the overall efficiency, and $A_{\text {phys }}$ is the physical collecting area.

The signals are read from the source position in the reconstructed dirty images with different integration times, with the source position determined from the final image. A CLEAN (Hogbom 1974) procedure is applied to the inner $21.6^{\prime}$ box, which roughly corresponds to the FWHM of the primary beam. The cleaned signal at the source position is also recorded, while the residual noise is measured in the 1 deg image excluding the inner clean region.

Figure 7 shows that an efficiency $\eta=(0.36 \pm 0.04)$ 




FIG. 7.- The signal and noise plotted against effective integration time for our sample. The black open squares and the red solid line with error bars represent the signal measured in dirty and cleaned maps respectively and have been multiplied by -1 in the plot. The green dashed line shows a noise estimate from the cleaned maps (see 43.3$)$. The blue dotted line shows the expected noise level given the effective integration time, an overall system efficiency of 0.36 , and a system temperature of $90 \mathrm{~K} .10^{6}$ sec of effective integration on the horizontal axis corresponds to roughly 3.3 hours on-source obervation time. The final signal-to-noise ratios (SNR) in the cleaned images are 6.0 for A1689, 6.4 for A1995, 13.7 for A2142, 5.2 for A2163, and 6.6 for A2390).

is more representative for the current data, giving a point source sensitivity of $63 \pm 7 \mathrm{mJy}$ in 1 hour of onsource integration when the subtraction scheme is applied. Abell 2390 has a higher noise, and we believe this may be caused by the presence of point sources in the fov. Liu et al. (2009) investigate the contamination by point sources and the primary CMB.

\section{CONCLUSION}

To detect galaxy clusters with the AMiBA, we must achieve system stability on timescales of hours. We have optimized the performance of AMiBA by measuring and compensating for the delays between antennas, and using beam switching techniques to cancel out instrumental and environmental effects. Planet calibrations provided corrections for passband response. Overall efficiency for AMiBA was $\eta_{\text {all }}=0.36 \pm 0.04$, with a major loss from the antenna efficiency, $\eta_{\text {ant }}=0.58$.

Using a system temperature of $90 \mathrm{~K}$, an effective bandwidth of $5 \mathrm{GHz}$ per band, and an overall efficiency of
$0.36 \pm 0.04$, the point source sensitivity of AMiBA in 1 hour of on-source integration $\left(t_{\text {eff }}=302400 \mathrm{sec}\right)$ is found to be about $63 \pm 7 \mathrm{mJy}$ when the subtraction scheme is applied. The effective integration is about $60 \%$ of the on-source integration time. The loss of $40 \%$ of observing time is mostly due to lower weighting applied to some receivers or baselines experiencing hardware problems. There were very few observations made when the weather was not good in the 2007 and 2008 observing season.

The flux density error consists of the calibrator flux scale uncertainty of $\pm 5 \%$, and the cross-calibration error $\pm 3 \%$, which also includes the lag-to-visibility flux scale error of $\pm 2 \%$. The latter two errors are well below the thermal noise in all clusters observed during 2007 and 2008. Investigation of noise in cleaned images shows that longer integration, aimed at measuring primordial CMB fluctuation, will be promising.

The resulting successful detections of clusters have led to a number of scientific results including a measurement 
of the Hubble constant and the study of the hot gas distribution in the clusters. These are discussed further in the companion papers.

Acknowledgments Capital and operational funding for AMiBA came from the MoE and the NSC as part of the Cosmology and Particle Astrophysics (CosPA) initiative. Additional funding also came in the form of an Academia Sinica Key Project. Support from the STFC for $\mathrm{MB}$ is also acknowledged.

\section{REFERENCES}

Bond, J. R. et al. 2005, ApJ, 626, 12

Chen, M.-T. et al. 2009, ApJ, submitted

Goldstein, J. H. et al. 2003, ApJ, 599, 773

Griffin, M. J. et al. 1986, Icarus, 65, 244

Ho, P. T. P et al. 2009, ApJ, accepted (astro-ph/0810.1871)

Hogbom, J. A. 1974, A\&AS, 15, 417

Huang, C.-W. L. 2009, ApJ, submitted

Huang, Y.-D. et al. 2008, SPIE, 7012, 70122H

Koch, P. M. et al. 2009a, ApJ accepted

Koch, P. M. et al. 2009b, ApJ, submitted

Koch, P. M. et al. 2009c, in preparation

Kuo, C.-L. et al. 2004, ApJ, 600, 32

Li, C.-T. et al. 2004, SPIE, 5498, 455
Lin, K.-Y., Woo, Tak-Pong, Tseng, Yao-Huan, Lin, Lihwai, and Chiueh, Tzihong 2004, ApJ, 608, 1L

Lin, K.-Y. et al. 2008, SPIE, 7012, 701207

Liu, G.-C. et al. 2009, ApJ, submitted

Nishioka, H. et al. 2009, ApJ accepted (astro-ph/0811.1675)

Padin, S. et al. 2002, PASP, 114, 83

Page, L. et al. 2003, ApJS, 148, 39

Pearson, T. J. et al. 2003, ApJ, 591, 556

Umetsu, K. et al. 2009, ApJ accepted (astro-ph/0810.0969)

$\mathrm{Wu}$, J.-H. P. et al. 2009, ApJ accepted (astro-ph/0810.1015)

Ulich, B. L. 1981, AJ, 86, 1619 